\title{
Next Steps for Value Sensitive Design
}

\author{
Alan Borning \\ Dept. of Computer Science \& Engineering \\ University of Washington \\ Seattle, Washington, USA 98195 \\ borning@cs.washington.edu
}

\author{
Michael Muller \\ IBM Research \\ One Rogers Street \\ Cambridge, MA, USA 02142 \\ michael_muller@us.ibm.com
}

\begin{abstract}
Questions of human values often arise in HCI research and practice. Such questions can be difficult to address well, and a principled approach can clarify issues of both theory and practice. One such approach is Value Sensitive Design (VSD), an established theory and method for addressing issues of values in a systematic and principled fashion in the design of information technology. In this essay, we suggest however that the theory and at times the presentation of VSD overclaims in a number of key respects, with the result of inhibiting its more widespread adoption and appropriation. We address these issues by suggesting four topics for next steps in the evolution of VSD: (1) tempering VSD's position on universal values; (2) contextualizing existing and future lists of values that are presented as heuristics for consideration; (3) strengthening the voice of the participants in publications describing VSD investigations; and (4) making clearer the voice of the researchers. We propose new or altered approaches for VSD that address these issues of theory, voice, and reportage.
\end{abstract}

\section{Author Keywords}

Value Sensitive Design; participatory design; design; collaborative ethnography; qualitative research; universal values; culturally-specific values; feminism; voice

\section{ACM Classification Keywords}

K.4.1 [Computers and society]: Public policy issues Ethics;

\section{INTRODUCTION}

As the field of human computer interaction has matured, an increasing body of HCI research has concerned itself with human values. The ACM Digital Library shows a fivefold increase in papers mentioning "human values" during the past ten years, from 20 in 2000 to 113 in $2010, r=0.92$, $\mathrm{p}<.001$ (Figure 1). At the same time, a number of approaches for systematically considering human values in information technology have emerged, in particular Value

Permission to make digital or hard copies of all or part of this work for personal or classroom use is granted without fee provided that copies are not made or distributed for profit or commercial advantage and that copies bear this notice and the full citation on the first page. To copy otherwise, or republish, to post on servers or to redistribute to lists, requires prior specific permission and/or a fee.

CHI'12, May 5-10, 2012, Austin, Texas, USA.

Copyright 2012 ACM 978-1-4503-1015-4/12/05...\$10.00.
Sensitive Design (VSD) [28, 30]. VSD is "a theoretically grounded approach to the design of technology that accounts for human values in a principled and comprehensive manner throughout the design process" [30, p. 349]. The word "value" here is defined broadly, as "what a person or group of people consider important in life". Key features of VSD are a tripartite methodology, consisting of iteratively applied conceptual, empirical, and technical investigations; an emphasis on considering indirect as well as direct stakeholders (that is, people who are affected by a technical system but don't use it directly, as well as those who do); and an interactional theory of the relationship between values and technology.

VSD has been an important part of CHI, leading to many influential findings and analyses (for example, [16,28, $29,30,31,32,34,41,50,51,57,76,77])$. More recently, as VSD itself has matured, a number of critiques and suggestions for its evolution and further development have been published (e.g., $[1,3,48,63])$. This essay is intended as a contribution toward that evolution. The problem we engage is that, in our view, VSD as currently presented overclaims. One aspect of this overclaiming is that VSD takes a number of stances that are broader and more sweeping than are necessary, or even warranted, for the work it is doing. A second aspect is that there are a set of rhetorical moves in some of the writing about VSD that imply more authority on the part of the researcher than we believe is appropriate. We suggest that unease about these claims has in turn inhibited the more widespread adoption and appropriation of this valuable theory and methodology. Another goal of our essay is to bring VSD into further dialog with a set of other relevant theories and approaches in the $\mathrm{CHI}$ community.

Our suggested next steps concern the following four topics.

1) Universal vs. culturally-specific values. VSD takes the position that certain values are universally held, but that the ways that these values play out in different cultures

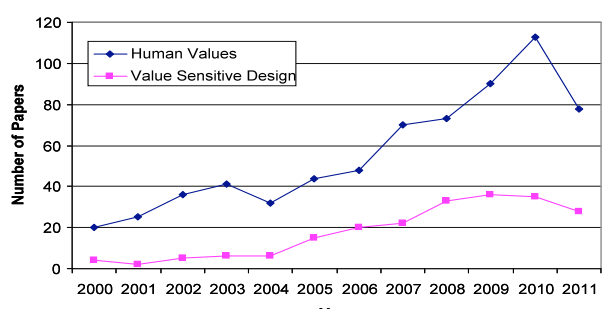

Figure 1. Increase in ACM interest in Human Values and Value Sensitive Design, 2000-2010. 
vary widely. Yet, as we discuss in more detail later, claims of universality for values are enormously problematic $[11,25,61]$. At the same time, a position of cultural relativism is problematic as well $[37,38,71]$. For this recommendation, avoiding overclaiming leads to a recommendation that VSD at the core should adopt a pluralistic (and perhaps more humble) position: simply that it doesn't have the answer to the difficult and longstanding question of universality, and further that a range of views on this question by different researchers can be accommodated under the VSD rubric. This pluralistic position would also help to clarify the question of whether VSD is a method that can be applied in principle to any set of values, vs. VSD as the methodological instantiation of a particular set of values (e.g., [1,63]).

2) Contextualizing and qualifying lists of values offered for consideration. A number of VSD papers have included lists of values, either as ones that may have a distinctive claim on resources in the design process, or as heuristics for suggesting values that might be implicated in a design. We acknowledge the utility of heuristic lists (while noting the potential problem of biasing the results of an investigation). However, we suggest that it would be better, when presenting such lists, to be explicit about the particular culture and viewpoint in which they were developed, rather than omitting this context and in the process making implicit claims about more universality than are warranted, or perhaps even intended.

3) Strengthening the voice of the participants in writing about (and perhaps in conducting) the VSD investigation. The publications of VSD have sometimes privileged the voice of the researchers in presenting qualitative results, not only as interpreters (a viable tradition in ethnography and qualitative analysis) but also as reporters (a more problematic strategy, if there are differences between the informants vs. the researchers [54]). The participatory design tradition has demonstrated the value and fidelity of informants speaking for themselves through $\mathrm{HCI}$ publications $[12,55,66]$. We believe there is overclaiming of knowledge and authority in the substitution of the researcher's voice for the voice of the informants. Participatory design also commits, in a substantive way, to co-design and sharing power between the researchers/designers and the users of the technology (see also [47]). While we do not go so far as to recommend that this be a core commitment of VSD as well, we do suggest that this should be considered carefully in VSD projects, and that in some cases such power sharing should be an explicit goal of the project.

4) Making more salient the voice of the researchers and designers themselves in writing about the investigation. Questions of values in HCI seldom have single, definitive, "objective" answers [43,44]. In many instances it may be useful for the reader if the researcher is more visible in the writing [17] - what is his or her background, relation to the participants in the study, and (for example if we are considering the role of the designer's own values) perhaps even relevant personal values $[24,78]$ ? As with our third suggestion, we see the overclaiming here as implying more authority and impartiality than is warranted, via the rhetorical move of using a disembodied voice, in areas in which the researcher's own culture and assumptions may limit exactly those qualities of authority and impartiality. Allied disciplines (ethnography, feminism) provide alternatives ways of reporting that appear convergent with the goals of VSD.

\section{BACKGROUND AND RELATED WORK}

There is a long history of work on values and technology, including early work by Mumford [56], Weiner [73], Weizenbaum [74], and Winner [75]. Regarding theory and methods that specifically address human values in design, either in the context of HCI or of information technology more generally, some notable examples in addition to VSD are work by Flanagan and Nissenbaum [26,27] and Cockton $[18,19]$. We include numerous additional references in context in the discussion that follows.

We now discuss in more detail our four suggested next steps in evolving VSD's theory and methods.

\section{UNIVERSAL VALUES IN ANALYSIS AND DESIGN}

Are there any values that are (or should be) universal for all humans, or are all values culturally or individually specific? This question, in one form or another, has exercised people for millennia, ranging from prophets to religious thinkers to philosophers to social scientists to people simply wondering what it means to be human or what is the nature of right conduct.

VSD's position on this question has evolved over the years. In an early paper on VSD, Friedman and Kahn [28] state:

When analyzing moral variability, conceptualizations of morality that entail abstract characterizations of justice and welfare tend to highlight moral universals, whereas definitions that entail specific behaviors or rigid moral rules tend to highlight moral cross-cultural variation. ... In our view, both questions [regarding moral universals vs. moral cross-cultural variation] have merit, and a middle ground provides a more sensible and powerful approach for the HCI community: One that allows for an analysis of universal moral values, as well as allowing for these values to play out differently in a particular culture at a particular point in time.

In a later paper, Friedman et al. [30] move from a philosophical to an empirical statement of a similar position:

VSD builds from the psychological proposition that certain values are universally held, although how such values play out in a particular culture at a particular 
point in time can vary considerably ... Note that this is an empirical proposition, based on a large amount of psychological and anthropological data, not a philosophical one. We also make this claim only for certain values, not all - there are clearly some values that are culture-specific.

There are huge problems with both the position that there are some values that are or should be universally held, as well as with the position that all values are either culturally or individually specific. On the one hand, the belief that there are universal values (from whatever source - God, evolutionary development, biological determinism, ...) has on occasion led to the further belief that a particular group, culture, or religion is the keeper of those values, and needs to impose them on others - with sometimes tragic consequences. Yet, if all values are culturally or individually specific, what gives one culture or group grounds to say that some practice of another culture (slavery, genocide, female genital mutilation, ...) is wrong (see [71])?

One response is to make this an empirical, scientific issue rather than a theological or philosophical one. (This is essentially the move made between the 2003 and 2006 papers referenced above.) In adopting this move, we still need to avoid the naturalistic fallacy (" 'is' does not equal 'ought' "), but this can be accomplished by qualifying prescriptive statements appropriately ("if we want to support human flourishing, then we should ..."). But a scientific approach to the issue of universal values is also problematic, given what we (the authors of this essay) view as past abuses, justified on "scientific" grounds. Notorious examples come from the literature on forced sterilization in service of a claimed value of genetic purity [65] or a claimed value of reducing mental illness [67]. More recently, social programs such as Head Start (a US government intervention to improve scholastic performance among poor and non-white children) have been endangered by claims that intelligence is a genetically-determined trait [42], and that academic enrichment programs should be targeted at the children who have the generic capacity to learn - a claimed value of using scarce resources where they will do the most good. See [70] for one of many critiques of this position.

A second response is to declare the researcher's position or commitment $[4,5,43,44]$, as is often the case in action research [40], collaborative ethnography [47], grounded theory [17], and participatory design $[12,13,55,66]$. But should such a position be one for the whole of VSD, i.e., one to which all VSD researchers must subscribe?

We suggest that VSD should take a more pluralistic position on this question of universal values, and simply say that this is a contested issue, to which VSD as such doesn't have the answer. If, in a particular study, it is important to come down on one side, then the researchers in that study should do so, and state their position, why they took it, and what the consequences were. We use the word "pluralistic" rather than "agnostic" in this suggestion, to accommodate exactly this. It should be possible for a researcher with a commitment to universal values - or with a commitment to all values being culturally constructed - to employ VSD. In practice, though, we suspect that the answer to this question has little if any impact on most actual projects. For example, even if there is some universally held value of privacy, the fact that "even while living in an igloo, [Inuit] have conventions that ensure some forms of privacy; yet such forms of privacy are not maintained by separated rooms, as they are in most Western cultures" [30] would be unlikely to have an impact on the design of technology. In other words, even if there is some universal aspect to the value of "privacy" (whether just a sliver or perhaps more), how this value plays out will be sufficiently different in the spectrum of human cultures that a universal design (whether of a social networking site or of a dwelling place) that works for all cultures seems like a chimera.

We note several significant caveats regarding this recommendation. First, universal values and universal design are distinct concepts, and indeed, as argued above, even if some values are universally held, this would not imply that universal designs were feasible. And VSD theory, even as currently expressed, does not imply that universal design is a realizable goal ("how such values play out in a particular culture at a particular point in time can vary considerably" [30]). Second, our reservations regarding the concept of universal values do not imply that we argue against cross-cultural studies that include looking carefully for commonalities as well as differences. (See [31] for an excellent example of this.) Quite the contrary: such studies become increasingly valuable as the global nature of the IT industry continues to develop and interfaces designed in one cultural context are used - and appropriated and molded - in another.

Finally, this is definitely not to say that the existence or not of universal values is an inappropriate topic for religion, philosophy, psychology, or anthropology, or that HCI researchers should not understand this thinking and research, perhaps deeply. Again, we suggest that this issue is one that VSD as such simply doesn't need to take a position on, at least given our current understanding of the issue from philosophy and various social sciences. Holding fast to VSD's position as described in [30] - or for that matter switching to a position of cultural construction of values - doesn't help advance the development and application of VSD. Rather the opposite: disagreements or simply unease within the research community about the position VSD has taken on this question have, we would argue, significantly impeded its development and application.

\section{WHOSE VALUES?}

Should VSD single out certain values as particularly worthy of consideration? If so, who chooses them, and how should they be chosen? VSD's position on this question has also 
evolved over the years. In an early chapter, Friedman and Kahn [28] write: "We review and discuss 12 specific values with ethical import. Some of these values have garnered individual chapters in this handbook. But, by including these values here, we highlight their ethical status and thereby suggest that they have a distinctive claim on resources in the design process." The chapter then goes on to list and discuss these values: Human Welfare, Ownership and Property, Privacy, Freedom From Bias, Universal Usability, Trust, Autonomy, Informed Consent, Accountability, Identity, Calmness, and Environmental Sustainability.

They are also careful to note that "this list is not comprehensive. Perhaps no list could be, at least within the confines of a chapter. Peacefulness, compassion, love, warmth, creativity, humor, originality, vision, friendship, cooperation, collaboration, community, purposefulness, devotion, diplomacy, kindness, musicality, harmony-the list of other possible values could get very long very fast."

In a subsequent paper [30], the list of specific values (now expanded from 12 to 13 with the addition of Courtesy) is introduced as: "We stated earlier that while all values fall within its purview, Value Sensitive Design emphasizes values with ethical import... [W]e present a list of frequently implicated values... intended as a heuristic for suggesting values that should be considered in the investigation - it is definitely not intended as a complete list of human values that might be implicated." A key difference between the descriptions is that the list is now presented as a design heuristic (ask yourself, is this value implicated in my design?), rather than as values that the authors suggest "have a distinctive claim on resources in the design process".

The evolution in VSD toward evocative heuristics rather than lists that call out a specific set of values has continued, with a notable example being Envisioning Cards [34]. Nevertheless, we find the presentation of the lists in both the 2003 and 2006 papers problematic: it would have been better to contextualize them by noting that these are typical values from a liberal and relatively privileged Western perspective (and that have been evidenced in HCI work to date, which has usually been in done in such a context), rather than implicitly claiming more universality by presenting them without such qualification. We also note that the lists are in English, and use the English phrases for the concepts. Translations will not be precise, and may well bring out different issues. Indeed, this is noted in published VSD work, in particular a 2008 VSD crosscultural study on attitudes toward privacy in public spaces in the U.S. and Sweden [31]. That paper describes the difficulties of translating the word "privacy" into Swedish. The Swedish translation that the researchers adopted ("personlig integritet") encompasses both controlling information flow (as in the English language meaning) but also maintaining the quality (accuracy) of that information [31, p. 143].
A living theory and method will evolve, and a past description will not necessarily capture the current understanding. Or it is often easy in retrospect to see how something could have been said better. Nevertheless, an explicit qualification of the context and limitations of these early lists as suggested above would be useful.

Le Dantec et al. [48] raise a similar concern about what they view as an inappropriately privileged and prescriptive role for the list of values in the 2003 VSD paper [28]. However, we soften our own concern in three respects. First, we note that the list of values as presented in a later paper [30] is introduced as "a heuristic for suggesting values that should be considered in the investigation" rather than as values that "have a distinctive claim on resources in the design process". Second, one of the grounds for Le Dantec et al.'s concern is a claimed prescribed ordering for the investigations that comprise VSD's tripartite methodology: first conceptual, then empirical, then technical. Here we disagree: in doing value sensitive design one can start with any one of the investigations; and indeed, there are published VSD investigations that start with each of the three. For example, the early work on browser cookies and privacy [50] began with conceptual investigations, the Watcher and the Watched studies [29] began with empirical investigations, and the CodeCOOP work [51] began with technical investigations. Third, we observe that VSD, as a living theory and method, will and ought to evolve over time (one example of this being the continued evolution toward evocative heuristics rather than lists that call out a specific set of values), and so we don't want to make too much of a statement from a 2003 paper.

Should VSD as such have any heuristic lists of values that might arise in particular applications? What about more evocative and less specific heuristics such as Envisioning Cards? One position is that any lists, or even other kinds of heuristics, are harmful, in that they tend to bias the researcher or designer toward existing analyses and ways of thinking. (Indeed, the authors in [48] seem to lean toward such a position, at least as regards lists.) In our view, this position is rooted in a valid concern, but one that well-done empirical investigations help mitigate. However, in any case, taking such a purist stance in all investigations would make VSD not a practical method in many situations, e.g., in an industrial setting with a tight schedule. For example, when designing a new privacy options interface for a social media application, it seems unreasonable to tell the designers not to look at some careful VSD work on the privacy options tool for a different social media application, but to start from scratch. More broadly, we think there is benefit in having heuristics and cues (including lists of values that might be implicated) to aid designers in considering values that may be at stake in a given system. Certainly such lists of values or other tools should be contextualized with information about who wrote it, in what milieu, and for what purpose. Using a variety of heuristics and suggestions about values that might be present seems likely to improve 
rather than diminish the odds of not overlooking important values, and consequently the quality of the end result.

An essential activity in broadening VSD is to continue to build up collections of case studies, heuristics (and yes, even lists) that are particularly relevant for a broad range of cultures and contexts. One omission (notable given the long history of participatory design in $\mathrm{HCI}$ ) is labor concepts, such as solidarity and collective action, as well as some relational values [8]. In terms of other cultures, Alsheikh et al. [3], for example, provide a case study situated in Arab cultures and values.

Finally, consider values in domains not typically seen in publications at $\mathrm{CHI}$ or $\mathrm{CSCW}$, for example, military values. There is published work in this area: Cummings [21] describes a VSD case study (part of a proposed engineering curriculum) of the hypothetical development of a command and control supervisory interface for a military cruise missile. The conceptual analysis is built around the value of human welfare, which is already often seen in VSD work - but here drawing on different philosophical traditions, namely the theory of just war, and the principles of proportionality and of discrimination. One can also imagine VSD analyses for military applications for direct use by combat soldiers or other warfighters that implicate additional values, such as courage, honor, discipline, loyalty, and accountability. This domain does not fit comfortably in the usual values discussion in the HCI community - but (unless one believes there should be no new military systems) it makes sense to consider military systems from a VSD perspective and to design them well, so these philosophical traditions and values are certainly relevant (e.g., [60]).

A related question is whether VSD should itself substantively commit to certain values, for example, in the way that participatory design substantively commits to the values of participation and democracy, that collaborative ethnography commits to co-authorship as well as co-analysis with the affected population [47], or that action research commits to emancipatory goals [40]. If so, how are those values chosen and who does so? We (the authors of this essay) tread cautiously here. Tempting as it is to include values we personally hold dear, we suggest that VSD commit to just those values that are essential to making VSD itself work: namely, pluralism or inclusivity (necessary to do the analysis of direct and indirect stakeholders well), plus openness and transparency (values that are important in many other methods as well of course, not just VSD).

Regarding other values beyond these, we would underscore and build on the distinction among explicitly supported values, stakeholder values, and designer values [16]. These are respectively values that are explicitly supported and adopted in a given investigation or project, values that are important to some (but not necessarily all) stakeholders, and the designer or researcher's own personal values. Then, for example, if researchers wanted to adopt participatory design values (and practices) in the course of a
VSD project, they would make those PD values be explicitly supported values, drawing on PD methods as appropriate as well; and similarly for researchers wanting to adopt values and practices from the collaborative ethnography or action research traditions. Researchers in the PD, collaborative ethnography, and action research traditions derive their values explicitly from their coresearchers (community members), as well as those who have gone before them. As experience is built up with VSD projects adopting a given set of explicitly supported values, the value and stakeholder analyses and guidance regarding methods can be shared and co-evolved. We acknowledge that this is a less satisfactory situation for VSD, from the point of view of shared and co-constructed sets of community values, since those values would be not an integral part of VSD itself - but short of VSD substantively committing to a wider collection of values, it may be a useful approach.

A further elaboration of this idea would be for VSD researchers or practitioners to enact a form of democratic voicing, by creating a website for these lists. In one of many possible designs, a researcher could contribute a list of values, and other people could comment on that list. If a second person wanted to propose a variation, s/he could make a (clearly linked) copy of the list, and rewrite selected aspects. Discussion comments might then be linked back to both lists. In this proposal, VSD would support pluralistic values with pluralistic lists, and would provide an example of democratic methods by encouraging lively discussions about each list and its values.

\section{THE VOICE OF THE PARTICIPANTS}

There are two aspects to our third suggestion, which regards the voice of the participants.

The first aspect is a relatively contained one, regarding hearing directly the voices of the participants. In presenting work about different users or stakeholders, many of the disciplines that meet in $\mathrm{CHI}$ and $\mathrm{CSCW}$ favor hearing the voices of the users or stakeholders. This point would seem to be particularly important for work related to values $[41,51,57,58,76]$. For readers with a background in ethnography or cultural critique, this is a crucial point. It is, in the influential essay by Alcoff, "the problem of speaking for others" [2]. When we interpose ourselves between an informant and an audience (as most researchers must do), there is the risk of unintentional ventriloquism - i.e., stating the researcher's own views as if those views had been articulated by the informants [52]. The common solution to this problem is to develop disciplines of data collection, analysis, and reporting that bring the informants' words to the reader with some assurance of directness and fidelity. Judicious verbatim quotation, with good contextualization, is the most frequent response to this problem in HCI and CSCW. The point is not to guard against some "hidden agenda" on the part of the researcher. The risk is that everyone is capable of unintentional, well-meaning 
ventriloquism, and so we invoke these disciplines in order to protect ourselves against doing something we would very much regret.

The second aspect of our suggestion is less well-worked out, and is indeed in many ways a direction for future research. VSD originated in part in trying to apply participatory design in contexts for which PD methods at the time were ill-suited due, for example, to a lack of a clear organizational boundary, such as applying PD methods to browser design [50], or in today's world, Facebook. (Also see [15].) However, in the process, some of PD's core commitments to co-design and sharing power were weakened, and instead power is back in the hands of the researchers.

In the decades since PD was first developed, it has evolved to include different stakeholder groups (children, elderly, disabled, ...), as well as more situations with less clear organizational boundaries; and approaches to adapting it to such contexts have begun to emerge [54]. We suggest that the traditional PD commitments to co-design and power sharing be carefully considered in VSD projects as well, taking advantage of this newer work. In some cases, it will be appropriate to make co-design and power sharing be explicitly supported values for the project. At the same time, we should continue to investigate ways to support these values with more diverse stakeholder groups. (Davis [22] discusses some of these relations between VSD and $\mathrm{PD}$ in the context of persuasive technologies.)

These considerations allow us to reframe some of the VSD critiques raised in [48]. We earlier disagreed with Le Dantec et al.'s claim that VSD prescribes a particular order of investigations. We would in fact also disagree with their claim that they started with an empirical investigation. In their work with homeless people, we suggest that they actually started with a conceptual investigation, not an empirical one: namely, they analyzed the power relationships and decided that they should talk with the homeless people. There is otherwise not an a priori reason to talk with homeless people, rather than service agency staff, police, business owners, or other stakeholders. ${ }^{1}$ But despite these two disagreements, at yet another level we believe the researchers got something very right: by giving voice to the homeless people, they foregrounded the values and concerns of a group that is otherwise often disempowered and silenced (see also [4,40,47,52]).

Le Dantec et al. also highlight the photo-elicitation interview (PEI) method as an empirical method: "The PEI works well when there is a significant power differential

\footnotetext{
${ }^{1}$ Or if this decision were not formally part of a conceptual investigation, the researchers are still deciding what to attend to, which strongly shapes the VSD investigation. Deciding to talk with the homeless people doesn't come out of nowhere.
}

between researcher and subject by shifting the power dynamic toward the participants by letting them shape the direction of the interview through the content they have created" (see also [55,59,72]). We would echo the importance of attending to power differentials, but re-frame this recommendation as being about giving voice to the participants in the VSD study rather than prescribing particular methods. (Just as part of VSD's theory is an interactional theory that says technologies have value suitabilities, in the same way we suggest that empirical methods have different value suitabilities - whether or not photo-elicitation is in fact a more appropriate empirical method here, we can still frame Le Dantec et al.'s recommendation as being about value suitabilities for different empirical methods.)

\section{THE VOICE OF THE RESEARCHERS}

Our last recommendation for evolving VSD is also tied to voice, this time the voice of the researchers and designers. Questions of values seldom have single, definitive, "objective" answers. In many instances it may be useful for the reader if the researcher is more visible in the writing what is his or her background, relation to the participants in the study, and perhaps even relevant personal values [17]? This practice of self-disclosure is standard for example in writing ethnographies $[23,24]$. There are now examples of this in the VSD literature as well, and we suggest expanding the practice.

As an existing example, Alsheikh et al. [3] discuss how the authors' backgrounds impact theory and method: "While these technologies support full video, we used an audioonly connection, as the first author, an Iraqi citizen who studied in Egypt, recognized an Arabic cultural taboo associated with discussing relationships and intimacy", or "Close readings of the data, and a careful consideration of what we, as researchers coming from Iraq, the US, and the UK, took for granted in our own notions of privacy, alerted us to the need for some alternative theoretical lenses with which to interpret it." In this example, the researchers' experience allowed a better method of inquiry.

More broadly, there are claims that the perspective or "standpoint" of the researcher can become both a powerful tool of inquiry (i.e., certain life experiences provide insights that can inform an exploration), and a set of limits on what the researcher is able to perceive and understand [38]. Applying this tradition to a VSD project to improve the safety of homeless young people, Woelfer et al. [77] include a section entitled "Researcher Stance", and write: "The research team is comprised of people with backgrounds in HCI, design, and security. We work from a design stance that seeks to both understand the situated context as well as to create meaningful change. ... On reflection, we have also found that this work changes us, especially how we apprehend the U-District [neighborhood] and think of the young people we have met in research and later encounter on the street. In these public encounters, we 
are trained to acknowledge the person but subtly, so as not to reveal our inter-personal familiarity to bystanders."

Another facet of this recommendation is connected with the aforementioned distinction among explicitly supported values, stakeholder values, and designer values. If researchers make this distinction in a given project, it may well be useful for the reader to know what are the relevant personal values of the researchers or designers, as well as the values that are explicitly supported in that project and the relevant values of the key stakeholders, so that the reader can judge how well the different kinds of values are distinguished and treated in the work [37]. This kind of clarity is particularly important if there were value tensions or conflicts among values in these three groups. Our earlier proposal for self-disclosure by authors is also important here, in the event that there might be values conflicts between authors and the people they are describing [52]. Similarly, knowing who is funding the work (and what goals they might have for it) may be important for the reader in evaluating the research.

A final facet involves subtle but arguably important changes in wording and emphasis, to make it clearer that there is a person or group taking the role of researcher, of working through a method, interviewing participants, analyzing data, and so on - avoiding the disembodied "view from nowhere" [36]. It is common in CHI and CSCW for the author to take the role of analyst and/or designer. Indeed, part of the motivation of PD is to share the responsibility and power to design (e.g., [12]) as well as to interpret and to analyze (e.g., [53] - see also [47]). We submit that questions of values and ethics would benefit from a similar sharing of responsibility and power - i.e., to perform an analysis of values and/or ethics [55]. This sharing could be particularly important in inter-cultural situations, in which disagreements about values may stem in part from disagreements about the "first principles" that underlie the ethical positions [49] (e.g., the tragic disagreements between the Navajo and Hopi nations of North America, about what it means to live on the land, and what "empty" land is good for [9]). Thus, a more contextualized VSD method would include a description of the ethical analyses of the people in the situation - acting as professional or "lay" ethicists. Appropriate background and citations regarding the underlying ethical theory (e.g., deontological, utilitarian, ...), may also be useful and important.

For example, in discussing a principled prioritization scheme for deciding where to put resources, Friedman et al. [32] write: "Our strategy for prioritization thus serves as a check on allowing funding and other pragmatic considerations to unduly influence the implementation priorities." We suggest that this leaves invisible exactly who invokes this check (and perhaps who verifies that it was done in a principled fashion), and creates a subtle barrier, for example, to questioning how effective it was. We would rephrase this sentence as: "Our strategy for prioritization thus provides a tool that the designer - or a third party - can use as a check on allowing funding and other pragmatic considerations to unduly influence the implementation priorities." More words, indeed, but perhaps useful ones.

There are many methods for helping to make informants' voices heard. In addition to ethnographic quotations (e.g., $[20,68])$, the PD tradition has developed storytelling methods that can easily be adapted to include discussions of values and ethics, such as storyboarding methods to explicate work $[46,53,64]$, even in the absence of a common verbal language [69]; hypermedia for community storytelling [7]; community-produced photo-documentaries ("photo novellas") and video-documentaries to change government policy [72] or for urban design [45,59] and to document human rights violations [10]. Collaborative ethnography goes further, insisting that the report of a project should be understandable and critiqued in advance by the people who are being described, and should optimally be co-authored with them [47]. Recent VSD work has begun to use video in the field [33]; we suggest that video and other user-created media can also contribute to reports from the field to other researchers.

Woelfer and Hendry were careful to avoid direct quotations from their informants in a VSD project, as part of a principled plan to protect their informants' privacy [77]. The preceding examples from participatory methodologies show how informants can knowingly create documents (including multimedia, photographic, and video documents) that are intended as communications media to others. The explicit "audiencing" of these documents removes issues of quotation-without-permission, and can help VSD informants become advocates for their own perspectives, including their own values, and (if that is their goal) for the kinds of change that they want to bring about. We suggest that VSD can become a stronger method for both research and also social change through a strategy that enhances the voice of its informants (see [40,43,47]).2

\section{DISCUSSION AND PROPOSED NEXT STEPS FOR VSD}

VSD addresses issues of values experienced in people's lives. Like design issues, issues of values necessarily involve differences in perspectives, and often involve differences in power. Throughout this essay, in addition to our intended core contribution of a set of suggested next steps for VSD, we have endeavored to bring insights from other work in $\mathrm{CHI}$ and $\mathrm{CSCW}$ that bears on these kinds of

\footnotetext{
${ }^{2}$ We are not suggesting that VSD adopt social change as a core value. Rather, we again make use of the distinction among explicitly supported values, stakeholder values, and designer values: if social change is one of the explicitly supported values in a project, then strengthening VSD in this respect becomes an important consideration.
} 
differences. We summarize this other work, and the insights we have derived from it, in this section.

Practitioners of participatory design urged us to consider "users" as designers of their technology and of the work practices that may be reified in that technology [12]. Values are part of the background that participants bring to these collaborative settings. We have proposed that methods from participatory design, collaborative ethnography, and action research be used to inform the methods of VSD. We propose that VSD consider adapting those methods that foreground the needs, designs, and analyses of the "users" in participatory design, such as codesign, participant-led story-telling, drama, photodocumentary, and video-documentary (for a software methodology based on participatory design, see [13]; for a survey of methods, see [55]). An important topic for future research is to continue to investigate how to support participation and power-sharing in situations without clear organizational boundaries or groups of stakeholders.

Feminism provides additional lenses through which to consider questions of values and ethics [6]. Bardzell's recent analysis argued against "universal" statements and designs, and in favor of considerations of diversity of perspective, with a research focus on edge cases rather than on the relatively homogeneous middle of a population [4]. Feminist philosophies of science similarly advocate for the inclusion of diverse views, and for a form of "strong objectivity" in which each researcher's contribution cannot be evaluated for its claims until the researcher discloses her/his own position, perspective, and stakes in the work [37,38] (see more recently [39]). We have proposed that VSD can learn from feminist research methods in the sciences in general $[35,37,38]$, and in social science and HCI in particular (e.g. $[4,5,62]$ ), to include diverse voices, to examine the edge cases around a values-question [4], and to interrogate its own VSD practices with questions such as who is recognized as an analyst of values? (e.g., [55]).

HCI researchers in the post-colonialist domain can offer related critiques and insights (e.g., [44]). Post-colonialism is in part a critique of power, and can be applied to HCI power relationships among analysts and "users". We have proposed that, convergently with participatory design and with feminism, post-colonialism can help VSD to ask questions about "who is allowed to speak about whom?" (see also $[2,47]$ ) and "how are values-based decisions made and enacted when their impact is felt by people who are not recognized as design-makers or analysts?"

Similarly, some VSD projects are not only about reporting values issues and conundrums, but also about social activism. VSD researchers and practitioners in this area may find work in action research to be useful here [40]. This work could also be helpful in articulating the relationship between these two seemingly-related fields.
In more pragmatic or "operational" terms, we also think that VSD could benefit from a more democratic approach to its own methodology. VSD could use participatory technologies, such as social media, to build a rich and diverse set of resources. To cite just one example, the concept of lists of values has been controversial, and has evolved through VSD practice over the years (see the earlier section "Whose Values"?), and large gaps remain. We suggest that opening up the process to broader participation and sharing of power would be one useful step here - for example, VSD researchers could provide collaborative online tools to facilitate sharing, discussing, and refining research materials (e.g., lists, envisioning cards [34]) among the growing community of VSD practitioners. Forms of community-led VSD may emerge.

\section{ACKNOWLEDGMENTS}

A heartfelt thanks to Batya Friedman, for her leadership in developing Value Sensitive Design and leading the way for the past two decades, for a series of in-depth discussions about the ideas presented here over several years, and for cheerfully offering feedback on this paper. Many other members of the CHI and related communities have helped us develop these ideas over the years. Janet Davis, Dave Hendry, Lisa Nathan, and Jill Woelfer, and the anonymous CHI referees provided useful feedback on drafts. In the end, however, the authors bear sole responsibility for any errors or problems with these suggestions. This work was supported in part by the National Science Foundation under grant number CNS-0905384.

\section{REFERENCES}

1. Albrechtslund, A. Ethics and technology design. Ethics and Info. Tech. 9(1), 63-72, 2007.

2. Alcoff, L. The problem of speaking for others. Cultural Critique 20 (Winter 1991-1992), 5-32.

3. Alsheikh, T., Rode, J. and Lindley, S. (Whose) ValueSensitive Design? A Study of Long-Distance Relationships in an Arabic Cultural Context." Proc. CSCW 2011, ACM Press (2011), 75-84.

4. Bardzell, S. Feminist HCI: Taking stock and outlining an agenda for design. CHI 2010, ACM Press (2010), 1301-1310.

5. Bardzell, S. and Bardzell, J. Towards a feminist HCI methodology: Social science, feminism, and HCI. CHI 2011, ACM Press (2011), 675-684.

6. Bardzell, S., and Churchill, E., (eds.), Interacting with Computers, special issue on feminism, in press.

7. Beeson, I., and Miskelly, C. Dialogue and dissent in stories of community. Proc. PDC 2000. New York: CPSR.

8. Belenky, M., Clinchy, B., Goldberger, N., and Turule, J. Women's ways of knowing: The development of self, voice, and mind, Basic, 1987/1997.

9. Benedict, E. The wind won't know me: A history of the Navajo-Hopi land dispute. Knopf, 1992.

10. Best, M., Smyth, T., Serrano-Baquero, D., and Etherton, J. Designing for and with diaspora: A case study of work for the 
truth and reconciliation commission of Liberia. Ext Abstracts CHI 2010, ACM Press (2010), 2903-2918.

11. Bickel, B. Weapons of magic: Afghan women asserting voice via the Net. JCMC 8(2), 2003.

12. Bjerknes, G., Ehn, P., and Kyng, M. (eds.). Computers and Democracy: A Scandinavian Challenge. Avebury, 1987.

13. Bødker, K., Kensing, F., and Simonsen, J. Participatory IT design: Designing for business and workplace realities. MIT Press, 2004.

14. Bødker, S., Ehn, P., Sjögren, D., \& Sundblad, Y. Co-operative Design - perspectives on 20 years with 'the Scandinavian IT Design Model'. Proc. NordiCHI 2000.

15. Borning, A., Friedman, B., and Kahn, P. H., Jr. Designing for Human Values in an Urban Simulation System: Value Sensitive Design and Participatory Design. Short paper, Proc. PDC 2004, Toronto, July 2004.

16. Borning, A., Friedman, B., Davis, J. and Lin. P. Informing public deliberation: Value sensitive design of indicators for a large-scale urban simulation. Proc. ECSCW 2005, Springer (2005), 449-468.

17. Charmaz, K., \& Mitchell, R.G. Jr. The myth of silent authorship: Self, substance, and style in ethnographic writing. In Hertz, R. (ed), Reflexivity and Voice, Sage, 1997.

18. Cockton, G. Value-centred HCI. Proc. NordiCHI'04, Tampere, Finland, 2004, 149-160.

19. Cockton, G. Getting there: Six meta-principles and interaction design. Proc CHI 2009, ACM Press (2009), 2223-2232.

20. Crabtree, A. Ethnography in participatory design. Proc. PDC 1998, Seattle, CPSR.

21. Cummings, M. Integrating ethics in design through the valuesensitive design approach. Science and Engineering Ethics 12(4), 701-715, 2006

22. Davis, J. Design methods for ethical persuasive computing. Persuasive '09, ACM Press (2009).

23. Dourish, P. Implications for design. Proc. CHI 2006.

24. Dourish, P. Ethnography. Tutorial presented at the Human Computer Interaction Consortium (HCIC), Winter Park, CO, Feb. 2010.

25. Ess, C., and Sudweeks, F. On the edge: Cultural barriers and catalysts to IT diffusion among remote and marginalized communities. New Media \& Soc, 3(3), 259-269, 2001.

26. Flanagan, M., Howe, D., and Nissenbaum, H. Values at play: Design tradeoffs in socially-oriented game design. CHI 2005, ACM Press (2005), 751-760.

27. Flanagan, M., Howe, D., and Nissenbaum, H. Embodying values in technology: Theory and practice. In J. van den Hoven and J. Weckert (eds.), Information Technology and Moral Philosophy. Cambridge University Press, Cambridge, UK, 2008, 322-353.

28. Friedman, B., and Kahn, P. H., Jr. (2003). Human values, ethics, and design. In J. A. Jacko and A. Sears (eds.), The Human-Computer Interaction Handbook, 1177-1201. Mahwah Erlbaum.

29. Friedman, B., Freier, N. G., and Kahn, P. H., Jr. Office window of the future? Two case studies of an augmented window. Ext Abstracts CHI 2004, ACM Press (2004), 1559.
30. Friedman, B., Kahn, P. H., Jr. and Borning, A. Value sensitive design and information systems. In P. Zhang and D. Galletta (eds.), Human-Computer Interaction and Management Information Systems: Foundations. M.E. Sharpe, Armonk, NY, 2006.

31. Friedman, B., Höök, K., Gill, B.T., Eidmar, L., Sallmander Prien, C., and Severson, R.L. Personlig integritet: A comparative study of perceptions of privacy in public places in Sweden and the United States. Proc. NordiCHI 2008, 142151.

32. Friedman, B., Borning, A., Davis, J. L., Gill, B. T., Kahn, Jr., P., Kriplean, T. and Lin, P. Laying the foundations for public participation and value advocacy: Interaction design for a large scale urban simulation. Proc 9th Annual International Conf. on Digital Government Research (dg.o2008), 305-314.

33. Friedman, B., Nathan, L.P., Lake, M., Grey, N.G., Nilsen, T.T., Utter, R.F., Utter, E.J., Ring, M., \& Kahn, Z. Multilifespan information system design in post-conflict societies: An evolving project in Rwanda. Ext. Abstracts CHI 2010, ACM Press (2010), 2833-2844.

34. Friedman, B., Nathan, L. P., Kane, S. and Lin, J. Envisioning Cards. University of Washington, Seattle, WA, 2011. Available at: http://envisioningcards.com.

35. Haraway, D.J. A cyborg manifesto: Science, technology, and socialist-feminism in the late twentieth century. In Simians, cyborgs and women: The reinvention of nature. Routledge, New York, 1991.

36. Haraway, D.J. Modest_Witness@Second_Millennium. FemaleMan (C_Meets_Oncomouse ${ }^{\mathrm{TM}}$ : Feminism and Technoscience. Routledge, New York, 1997.

37. Harding, S. The Science Question in Feminism, Cornell University Press, 1986.

38. Harding, S. (ed). The Feminist Standpoint Reader: Intellectual and Political Controversies, Routledge, 2003.

39. Harrison, S., Sengers, P., and Tatar, D. Making epistemological trouble: Third-paradigm HCI as successor science. To appear in Interacting with Computers.

40. Hayes, G.R. The relationship of action research to humancomputer interaction. TOCHI 18(3), 2011, 15:1-15:20.

41. Hendry, D.G., and Friedman, B. Theories and practices of design for information systems: Eight design perspectives in ten short weeks. Proc. DIS 2008, ACM Press (2008), 435-444.

42. Herrnstein, R.J., \& Murray, C. The Bell Curve: Intelligence and Class Structure in America, Free Press, 1994.

43. Hourcade, J.P., Bullock-Rest, N.E., Friedman, B., Nelson, M., Shneiderman, B., and Zephiris, P. HCI for peace: From idealism to concrete steps. Proc CHI 2011, ACM Press (2011), 613-616

44. Irani, L., Vertesi, J., Dourish, P., Philip, K., and Grinter, R.E. Postcolonial computing: A lens on design and development. CHI 2010, ACM Press (2010), 1311-1320.

45. Isomursu, M., Kuutti, K., and Väinämö, S. (2004). Experience clip: Method for user participation and evaluation of mobile concepts. Proceedings of PDC 2004. Toronto: CPSR.

46. Lafreniére, D. (1996). CUTA: A simple, practical, and lowcost approach to task analysis. Interactions 3(5), 35-39. 
47. Lassiter, E.L. The Chicago Guide to Collaborative Ethnography, University of Chicago Press, 2005.

48. Le Dantec, C.A., Poole, E.S. and Wyche, S.P. Values as lived experience: Evolving Value Sensitive Design in support of value discovery. Proc CHI 2009, ACM Press (2009), 11411150 .

49. Leidner, D.E., and Kayworth, T. A Review of Culture in Information Systems Research: Toward a Theory of Information Technology Culture Conflict. Mgmt. Info. Sys. Quart. 30(2), 2006.

50. Millett, L., Friedman, B., and Felten, E. Cookies and web browser design: Toward realizing informed consent online. Proc. CHI 2001, ACM Press (2001), 46-52.

51. Miller, J., Friedman, B., Jancke, G., and Gill, B. Value tensions in design: The value sensitive design, development, and appropriation of a corporation's groupware system, Proc. GROUP 2007, ACM Press (2007), 281-290.

52. Muller, M. Ethnocritical heuristics for reflecting on work with users and other interested parties. In M. Kyng and L. Mathiassen, Computers and Design in Context, MIT Press, Cambridge, MA, 1997.

53. Muller, M.J., and Carey, K. Design as a minority discipline in a software company: Toward requirements for a community of practice. Proc. CHI 2002, ACM Press (2002), 383-390.

54. Muller, M. Revisiting an ethnocritical approach to HCI: Verbal privilege and translation. In T. Erickson \& D. McDonald (eds.), HCI remixed: Reflections on notable HCI papers, MIT Press, 2007.

55. Muller, M. and Druin, A. Participatory design: The third space in HCI. In J. Jacko (ed.), The Human-Computer Interaction Handbook ( $3^{\text {rd }}$ edition), Mahwah, NJ: Lawrence Erlbaum Associates, in press.

56. Mumford, L. Technics and Civilization. University of Chicago Press, 2010. (Originally published 1934, Harcourt, Inc.)

57. Nathan, L.P., Friedman, B., Klasnja, P., Kane, S.K., and Miller, J.K. Envisioning systemic effects on persons and society throughout interactive system design. Proc. DIS 2008, ACM Press (2008), 1-10.

58. Nathan, L.P., Lake, M., Gray, N.C, Nilsen, T., Utter, R.F., Utter, E.J., Ring, M., Kahn, Z., \& Friedman, B. Multi-lifespan information system design: Investigating a new design approach in Rwanda, Proc. iConference 2011, ACM Press (2011), 591-597.

59. Patton, J.W. (2000). Picturing commutes: Informant photography and urban design. Proc. PDC 2000. New York: CPSR

60. Pew, R.W., and Mavor, A.S. Human-system Integration in the System Development Process: A New Look, National Academies Press, 2007.

61. Piecowye, J. Habitus in transition? CMC use and impacts among young women in the United Arab Emirates. JCMC $8(2), 2003$.
62. Rode, J. A theoretical agenda for feminist HCI. To appear in Interacting with Computers.

63. Saab, D.J. An ethnorelative framework for information system design. Proc. Fourteenth Americas Conference on Information Systems, Toronto, 2008.

64. Sanders, E. B.-N. Generative tools for co-designing. Proc. CoDesigning 2000. London: Springer.

65. Scales-Trent, J. Racial purity laws in the United States and Nazi Germany: The targeting process, Human Rights Quart. 23(2), 260-307, 2001.

66. Schuler, D. and Namioka, A. (eds.). Participatory Design: Principles and Practices. Lawrence Erlbaum Associates, Hillsdale, NJ, USA, 1993

67. Stubblefield, A. "Beyond the pale": Tainted whiteness, cognitive disability, and eugenic sterilization. Hypatia 22(2), 163-181, 2007.

68. Suchman, L., and Trigg, R. Understanding practice: Video as a medium for reflection and design. In J. Greenbaum and M. Kyng (eds.), Design at work: Cooperative design of computer systems. Erlbaum, Hillsdale NJ USA, 1991.

69. Tschudy, M.W., Dykstra-Erickson, E.A., and Holloway, M.S. PictureCARD: A storytelling tool for task analysis. Proc PDC'96, CPSR, Cambridge MA USA, 1996.

70. Wahlsten, D. The malleability of intelligence is not constrained by heredity, in Devlin, B., Feinberg, S.E., Resnick, D.P., \& Roeber, K. (eds), Intelligence, Genes, and Success: Scientists Respond to The Bell Curve. Springer-Verlag, 1997.

71. Walker, S., and Dearden, A. Designing for civil society. Interacting with Computers 17(1), 2005, 1-8.

72. Wang, C., Burris, M.A., and Ping, X.Y. Chinese village women as visual anthropologists: A participatory approach to reaching policymakers. Social Science and Medicine 42 (10), 1996, 1391-1440.

73. Weiner, N. The Human Use of Human Beings: Cybernetics and Society. Houghton Mifflin, Boston, 1954.

74. Weizenbaum, J. Computer Power and Human Reason: From Judgment to Calculation. W.H. Freeman, San Francisco, 1976.

75. Winner, L. Do artifacts have politics? Daedalus 109(1), Winter 1980, 121-136.

76. Woelfer, J.P., and Hendry, D.G. Homeless young people's experiences with information systems: Life and work in a community technology center. Proc. CHI 2010, ACM Press (2010), 1291-1300.

77. Woelfer, J.P., Iverson, A., Hendry, D.G., Friedman, B. and Gill, B. Improving the safety of homeless young people with mobile phones: Values, form and function. Proc CHI 2011, ACM Press (2011), 1707-1716.

78. Wyche, S.P. Investigating religion and computing: A case for using standpoint theory in technology evaluation studies. $\mathrm{PhD}$ dissertation, Georgia Institute of Technology, 2010. 